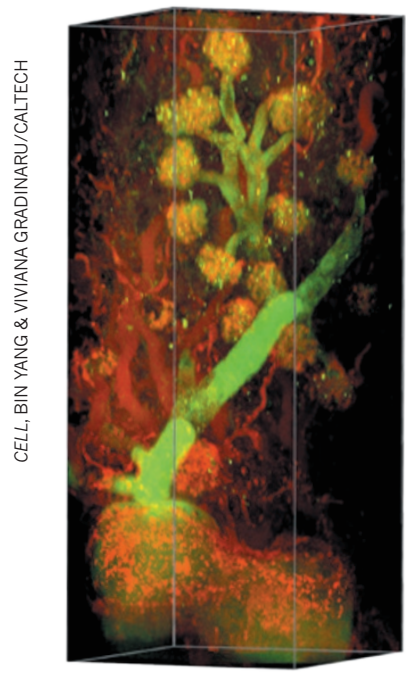

making tissue transparent have tended to work only on specific organs, such as the brain. A team led by Viviana Gradinaru at the California Institute of Technology in Pasadena tweaked an existing technique that stabilizes tissue and strips out light-blocking lipids with a cocktail of chemicals pumped through a dead rodent's circulatory system. Crucially, the process maintained the integrity of mouse neurons, kidney structures (pictured) and other tissue.

After one week for mice and two for rats, the brain and internal organs were clear and could be imaged under a microscope. The technique could allow researchers to see connectivity between the brain and other organs. Cell http://doi.org/tzz (2014)

Manta rays
change colour

Csilla Ari of the University of South Florida in Tampa observed five manta rays at the Atlantis Aquarium in the Bahamas, and discovered white markings appearing and disappearing in the space of a few minutes on their backs, fins and heads.

The changes seemed to occur in response to feeding or interaction with other manta rays, and may represent a form of communication. Two of the animals were giant manta rays (Manta birostris; one of two currently recognized species), and the other three may belong to a possible third species, which is similar but distinct from $M$. birostris.

Body coloration is used to identify species and individual rays, so the author says that understanding these colour changes is essential.

Biol. J. Linn. Soc.

http://doi.org/txc (2014)

\section{ASTROPHYSICS}

\section{Best gauge of exoplanet size}

Astronomers have made the most precise measurement so far of an exoplanet's size - for Kepler-93b, which orbits a star around 100 parsecs away.

Sarah Ballard at the

University of Washington in Seattle and her colleagues estimated the planet's diameter at about 18,800 kilometres

(1.48 times that of Earth), plus or minus 240 kilometres.

They used NASA's

Kepler space telescope to monitor seismic activity inside the planet's host star. They also used the Spitzer Space Telescope to observe Kepler-93b as it transited the star, applying a technique that ensured that for each measurement, light from the star fell on the centre of the same pixel in Spitzer's camera.

This allows for precision measurements of exoplanets' radii and masses, and even of the structure of their parent stars, the authors write.

Astrophys. J. 790, 12 (2014)

\section{ASTROPHYSICS}

\section{Novae join the $\gamma$-ray generators}

Astronomers have identified a previously unknown source of cosmic $\gamma$-radiation.

High-energy $\gamma$-rays are released in extremely energetic events such as pulsars and supernovae. But they were thought to be unlikely products of classical novae: explosions that occur on the surfaces of compact, burnt-out stars called white

SOCIAL SELECTION

\title{
Furore over genome function
}

Just how much of our genome serves a purpose? A recent study has reignited this debate on social media. After comparing the genomes of 12 different mammals (including humans, mice and pandas), researchers at the University of Oxford, UK, concluded that only about $8.2 \%$ of the human genome is shaped by natural selection. The rest, they argue, is non-functional. Observers noted the large difference between this estimate and a previous claim by the ENCODE (Encyclopedia of DNA Elements) Project that $80 \%$ of the genome is biochemically active. Patrik D'haeseleer, a computational biologist at Lawrence Livermore National Laboratory, California, tweeted: "Only between $8 \%$ and $80 \%$ of human \#genome is functional. Glad we've got that sorted out." At the heart of the issue are differing definitions of 'function'. Erick Loomis, an epigeneticist at Imperial College London, tweeted: "Maybe we should stop using 'functional' if we can't find a common definition." PLoS Genet. 10, e1004525 (2014)

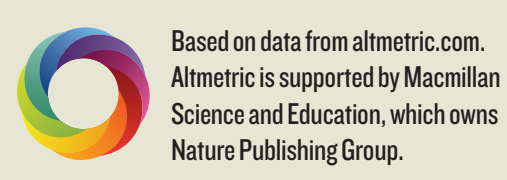

ONATURE.COM For more on popular papers: go.nature.com/boevfo dwarfs as they collect material from their neighbours in the binary system. The only nova previously seen emitting such rays came from an unusual type of star system. Now Teddy Cheung at the Naval Research Laboratory in Washington DC and his colleagues have used NASA's Fermi Telescope to detect high-energy $\gamma$-rays coming from three classical novae.

The otherwise unremarkable properties of the three stars suggest that such emissions could be common. It is not yet clear how particles surrounding the stars might be accelerated enough to produce the energetic radiation.

Science 345, 554-558 (2014)

\section{MICROBIOLOGY}

\section{A year with your microbes}

Microbial communities in the gut and mouth have been followed every day for an entire year. Stool and saliva samples collected from two men show that the communities remain fairly stable, but can be rapidly and broadly disrupted by events such as a bout of food poisoning or a holiday to a different continent.

Eric Alm, at the Massachusetts Institute of Technology in Cambridge, and his colleagues analysed these samples as well as health and lifestyle variables such as fitness, diet, exercise and mood recorded by the two volunteers.

One of the men developed food poisoning, which wiped out most of his gut bacteria; the microbes were eventually replaced with genetically similar species. And some lifestyle changes perturbed specific organisms - increasing dietary fibre, for instance, affected the abundance of $15 \%$ of the microbes in the gut. Genome Biol. 15, R89 (2014)

\section{$\rightarrow$ NATURE.COM}

For the latest research published by Naturevisit:

www.nature.com/latestresearch 